\title{
Targeting the microRNA-21/AP1 axis by 5-fluorouracil and pirarubicin in human hepatocellular carcinoma
}

\author{
Xiaodong $\mathrm{He}^{1, *}$, Jingjing $\mathrm{Li}^{1, *}$, Weidong Guo ${ }^{2, *}$, Wei Liu' ${ }^{1}$, Jia $\mathrm{Yu}^{3}$, Wei Song ${ }^{3}$, Lei \\ Dong $^{3}$, Fang Wang ${ }^{3}$, Shuangni $\mathbf{Y u}^{4}$, Yi Zheng ${ }^{1}$, Songsen Chen $^{3}$, Yan Kong ${ }^{5}$ and \\ Changzheng Liu ${ }^{3}$ \\ ${ }^{1}$ Department of General Surgery, Peking Union Medical College Hospital, Chinese Academy of Medical Sciences, Peking Union \\ Medical College, Beijing, PR China \\ ${ }^{2}$ Department of Hepatobiliary Surgery, The Affiliated Hospital of Medical College, Qingdao University, Qingdao, PR China \\ ${ }^{3}$ Department of Biochemistry and Molecular Biology, State Key Laboratory of Medical Molecular Biology, Institute of Basic \\ Medical Sciences, Chinese Academy of Medical Sciences, School of Basic Medicine Peking Union Medical College, Beijing, PR \\ China \\ ${ }^{4}$ Department of Pathology, Peking Union Medical College Hospital, Chinese Academy of Medical Sciences, Peking Union \\ Medical College, Beijing, PR China \\ ${ }^{5}$ Key laboratory of Carcinogenesis and Translational Research (Ministry of Education), Department of Renal Cancer and \\ Melanoma, Peking University Cancer Hospital \& Institute, Beijing, China \\ * Authors share co-first authorship \\ Correspondence to: Changzheng Liv, email: cz-liu@ibms.pumc.edu.cn
}

Yan Kong, email: k-yan08@163.com

Wei Liu, email: Liu_wei_95@sina.com

Keywords: microRNA-21, AP-1, hepatocellular carcinoma, chemotherapy, HIAC

Received: September 30, $2014 \quad$ Accepted: December 09, $2014 \quad$ Published: December 10, 2014

This is an open-access article distributed under the terms of the Creative Commons Attribution License, which permits unrestricted use, distribution, and reproduction in any medium, provided the original author and source are credited.

\section{ABSTRACT}

MicroRNAs function as oncomiRs and tumor suppressors in diverse cancers. However, the utility of specific microRNAs in predicting the clinical benefit of chemotherapy has not been well-established. Here, we investigated the correlation between microRNA-21 expression and hepatic arterial infusion chemotherapy with 5-fluorouracil and pirarubicin (HAIC) for hepatocellular carcinoma (HCC). We found that HCC patients with low microRNA-21 levels in tumors tended to have a longer time to recurrence and disease-free survival. We demonstrated that microRNA-21 suppression in combination with 5-fluorouracil and pirarubicin treatment inhibited tumor growth in subcutaneous xenograft mice models. Mechanistically, the AP-1 and microRNA-21-mediated axis was verified to be a therapeutic target of cytotoxic drugs and deregulation of this axis led to an enhanced cell growth in HCC. Taken together, our findings demonstrate that microRNA-21 is a chemotherapy responsive microRNA and can serve as a prognostic biomarker for HCC patients undergoing HAIC. Targeting microRNA-21 enhances the effect of chemotherapeutic drugs, thereby suggesting that microRNA-21 suppression in combination with HAIC may be a novel approach for HCC treatment.

\section{INTRODUCTION}

Hepatocellular carcinoma (HCC) is a primary liver cancer, the sixth most common cancer, and the third leading cause of cancer-related mortality worldwide [1-3]. Several effective biomarkers and new imaging techniques have enabled HCC detection at an early stage. However,
HCC patients still have a high rate of recurrence due to local invasion and intrahepatic metastasis, even after curative therapy [4]. The prognosis of patients with advanced HCC, where the tumor has spread across the liver or has invaded major vessels, remains extremely poor [4-5]. In these patients, conventional therapies using cytotoxic agents or novel targeted drugs have little 
clinical impact because of poor efficacy and possible complications [6]. Hepatic arterial infusion chemotherapy (HAIC) has several theoretical advantages such as a higher concentration of drugs delivered directly to the tumor and reduced systemic toxicity [7]. However, an optimal protocol of HAIC has not been established for HCC [8]. Therefore, it is imperative to identify useful biomarkers to predict the HCC clinical response to HAIC; these biomarkers might provide novel avenues of HAIC research and aid the early diagnosis and treatment of this highly malignant tumor.

Increasing knowledge of the tumor biology of HCC has elucidated a few coding and non-coding genes involved in hepatocarcinogenesis [9-12]. Although the fact that some of these deregulated genes have been validated as oncogenes or tumor suppressors, little is known regarding whether they can be potential predictors for HAIC. Previous studies have indicated that microRNA (miRNA) expression profiles or signatures can serve as diagnosis tools and predictors for diverse cancers [1316], and some of these deregulated miRNAs have been validated as oncomiRs or tumor suppressive miRNAs (ts-miRs) in hepatic tumorigenesis[17-18]. For example, miR-122, a specific miRNA in the liver, is downregulated in HCC tissues and acts as a ts-miR through negatively regulating cyclin G1 [19-20]. Various reports focusing on miR-21 have indicated a crucial role for this oncomiR in hepatocarcinogenesis [21-22] and demonstrate the extensive association of miR-21 with HCC prognosis and therapy [23-24]. Similarly, we have previously revealed that miR-21 functions as an oncomiR through suppressing the expression of PTEN, PDCD4, and RECK [21]. These findings imply that miR-21 is a candidate for HCC prognosis and therapy. However, several questions remain unanswered. For example, the correlation between miR-21 expression and the clinicopathological variables in HCC patients is unknown, and it is also unclear whether deregulated miR-21 expression in $\mathrm{HCC}$ is a response to HAIC. Chemotherapeutic drugs exert suppressive effects on HCC progression through regulating miR-21 expression.

Herein, we indentify miR-21 as a potential biomarker for predicting HAIC effects. we further demonstrate that chemotherapeutic drugs have clinical promise for HCC therapy through modulating a critical genetic pathway involving an miR-21-mediated program.

\section{RESULTS}

\section{miR-21 is a potential predictor of HAIC treatment for HCC patients}

Several studies have demonstrated that HAIC has clinical promise for HCC prevention and improves survival $[8,25]$. Because miR-21 has been validated as an oncomiR in hepatocarcinogenesis that leads to drug resistance $[21,24,26]$, we investigated whether its expression responded to HAIC treatment. In doing so, qRT-PCR analysis was conducted to evaluate miR-21 levels in 148 paired HCC samples, which indicated that miR-21 expression were upregulated in the majority of HCC specimens compared with the matched normal liver tissues (Figure. 1A, Figure. S1A). These data are consistent with our previous findings in 30 paired HCC tissues [21]. In-depth clinicopathological analysis was conducted on 109 patients with detailed information selected from the aforementioned HCC tissues, which showed that high miR-21 levels correlated with a more aggressive tumor phenotype $(P<0.001)$ and more extensive invasion $(P=0.03)$ (Figure. S1B-C; Supplementary Table $1)$. The relationship between miR-21 expression and variable pathological grade was also determined, and no significant differences among well, moderate, and poorly differentiated HCC tissues were found (Figure. S1D). A Kaplan-Meier analysis revealed that lower miR-21 levels were associated with longer disease-free survival (DFS) $(P=0.004$; Figure. S1E; Supplementary Table 2). Furthermore, we chose $67 \mathrm{HCC}$ patients undergoing HAIC with 5-fluorouracil and pirarubicin and conducted followup studies. These patients were divided into two groups based on recurrence in the 3 years after treatment. We observed that miR-21 expression was substantially higher in the tissues of HCC patients who recurred compared with those without recurrence (Figure. 1B). The Kaplan-Meier method also indicated that HCC patients with low miR-21 levels in tumors tended to have a longer DFS compared with those with high miRNA-21 expression, although the difference did not reach significance $(P=0.055$; Figure. $1 \mathrm{C}-\mathrm{i})$. We further conducted Kaplan-Meier analyses on patients with early stage HCC (pTNM stage I) and observed that higher miR-21 levels correlated with shorter DFS in early-stage HCC patients after HAIC ( $P=0.033$; Figure. 1C-ii). No significant difference between miR-21low and miR-21-high groups in HCC patients was found at advanced stages (pTNM stage II, III, and IV; $P=0.511$; Figure. 1C-iii). These findings suggest that HCC patients with lower miR-21 expression levels in tumors have a better response to HAIC treatment, especially at the early stages.

\section{miR-21 suppression combined with 5-fluorouracil and pirarubicin treatment inhibits HCC xenograft growth}

The above findings promoted us to further investigate whether 5-fluorouracil and pirarubicin treatment represented a better suppressive effect on tumor growth of HCC cells with miR-21 inhibition in vivo. To this aim, we conducted miR-21 suppression in 
Hep3b cells by using a lentiviral system (Lenti-miR-21-i) (Figure. S2A-C) and the subcutaneous xenografts were generated through inoculating the Hep3b cells infected with Lenti-miR-21-i and Lenti-scr, respectively. When the tumor reached a volume of about $100 \mathrm{~mm}^{3}$, chemotherapy was started and the tumor size and body weight were determined twice a week during treatment. We observed that miR-21 inhibition combined with 5-fluorouracil and pirarubicin treatment exerted a dramatically suppressive effect on the tumorigenicity of Hep3b cells in vivo, as compared to all other experimental groups (Figure. 2A-B; Figure. S2D). Moreover, we performed immunohistochemistry to detect the expression of $\mathrm{Ki}$ 67 in randomly selected xenograft mouse tumors, which demonstrated that the above treatment retarded HCC cell growth (Figure. 2C). These results supported our findings in $\mathrm{HCC}$ patients who receiving HIAC prevention with 5-fluorouracil and pirarubicin.
miR-21 expression is suppressed by 5-fluorouracil and pirarubicin in $\mathrm{HCC}$ cells

Based on the fact that HCC patients with low miR-21 levels in tumors had a better response to HAIC treatment with 5-fluorouracil and pirarubicin, we asked whether these two drugs offered a clinical benefit via down-regulating miR-21 expression. To this aim, we first evaluated the expression of miR-21 in several HCC cell lines using qRT-PCR (Figure. 3A), and the IC50 values of these drugs in Hep3b (miR-21-high) and SMMC7721 (miR-21-low) cells were determined. The estimated IC50 values for 5-fluorouracil and pirarubicin in the tested cell lines were as follows: Hep3b, 5-fluorouracil, $267.96 \mathrm{mg} / \mathrm{L}$; and SMMC7721, 5-fluorouracil, $131.38 \mathrm{mg} / \mathrm{L}$; Hep3b, pirarubicin, $1.65 \mathrm{mg} / \mathrm{L}$; SMMC7721, pirarubicin, 0.25 mg/L (Figure. S3A-B). Next, Hep3b and SMMC7721 cells
A

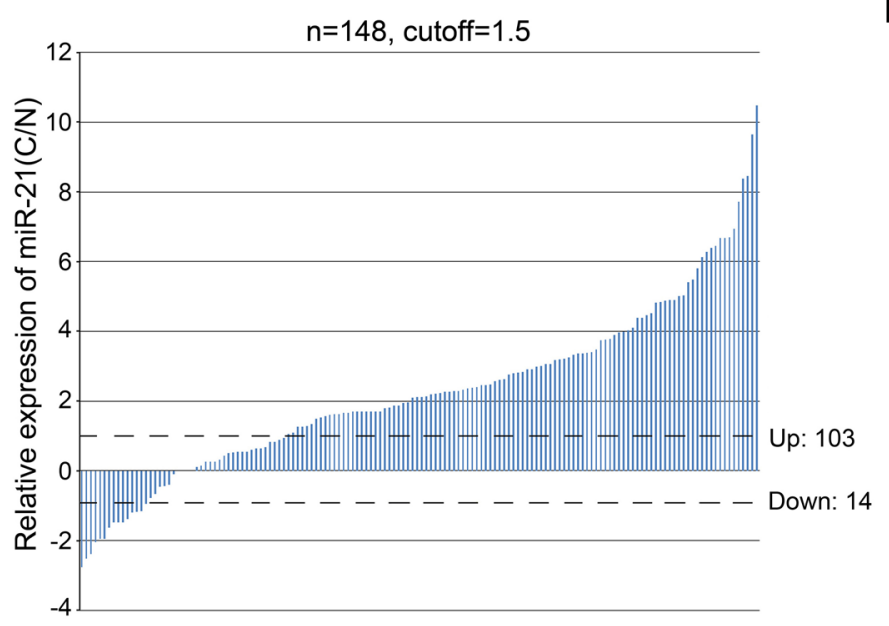

C

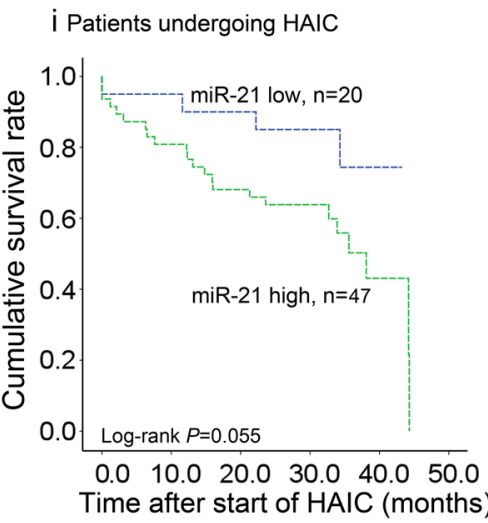

ii Patients at pTNM stage I

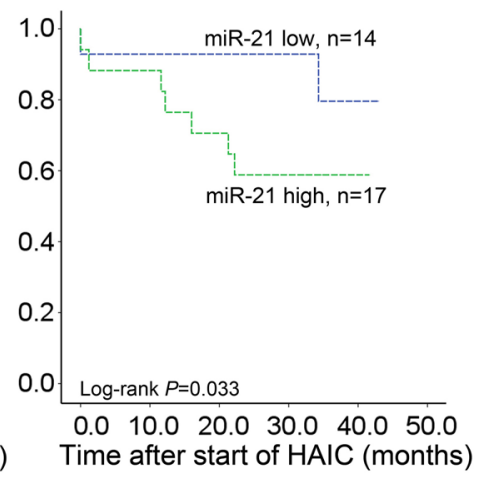

B

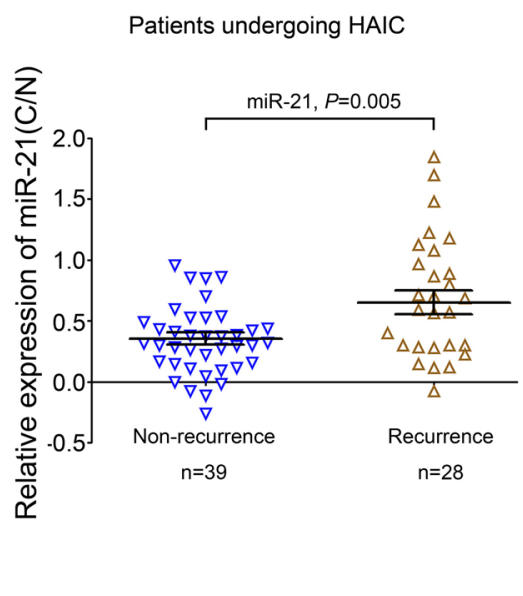

iii Patients at pTNM stage II-IV

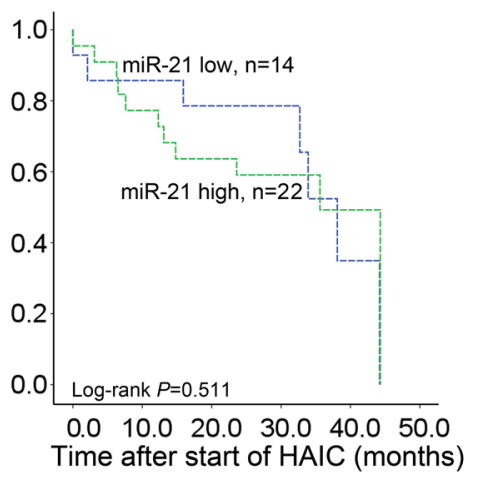

Figure 1: miR-21 is a potential predictor of HAIC treatment with 5-fluorouracil and pirarubicin for HCC patients. A: qRT-PCR analysis of miR-21 expression in 148 pairs of HCC tissues (C) and matched adjacent normal liver tissues (N). B: HCC patients with high levels of miR-21 tended to recur after HAIC treatment. C: Kaplan-Meier survival curves for DFS of HCC patients (i), HCC patients at the early stage (ii) and the advanced stage (iii) in relation to miR-21. Cut-off values for miR-21 (high/low expression) were determined through ROC analysis. 
were treated with 5-fluorouracil and pirarubicin, and qRTPCR analyses were performed. We observed that drugs treatment led to a significant decrease in miR-21 levels in the tested HCC cells compared with those treated with physiological saline (control) (Figure. 3B-C). We further investigated whether 5-fluorouracil and pirarubicin exerted their suppressive effect on miR-21 expression at the transcriptional level. qRT-PCR analyses were performed to evaluate the level of primary miR-21 (pri-miR-21) using three paired primers (up, cross, and down primers), which indicated a significant decrease in pri-miR-21 expression in 5-fluorouracil and pirarubicin treated Hep3b and SMMC7721 cells (Figure. 3D).

\section{5-fluorouracil and pirarubicin treatment lead to miR-21 downregulation through AP-1 proteins}

Previous studies have demonstrated that the AP-1 transcription factor family is involved in regulating miR21 expression [27-28]. Thus, we investigated whether the expression of AP-1 components was affected by 5-fluorouracil and pirarubicin. First, we tested the mRNA levels of c-Jun, JunB, and c-Fos using qRT-PCR analyses. Compared with control-treated cells, we observed that the expression of JunB and c-Fos was downregulated in 5-fluorouracil- and pirarubicin-treated HCC cells, and no obvious alteration in c-Jun mRNA levels was noticed (Figure. 4A-C). Further immunoblot analyses
A



B

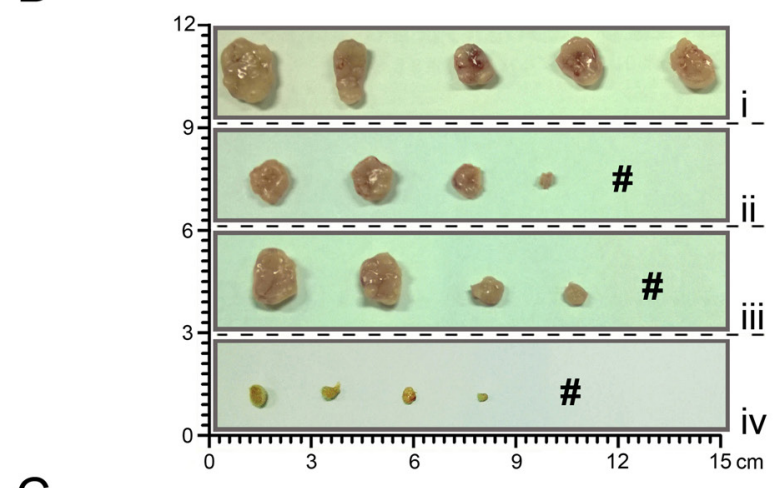

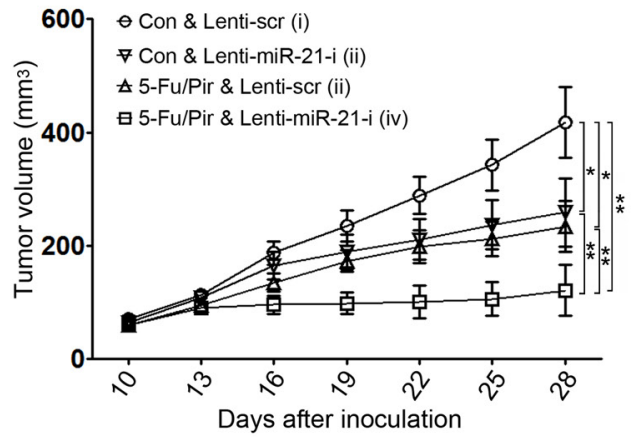

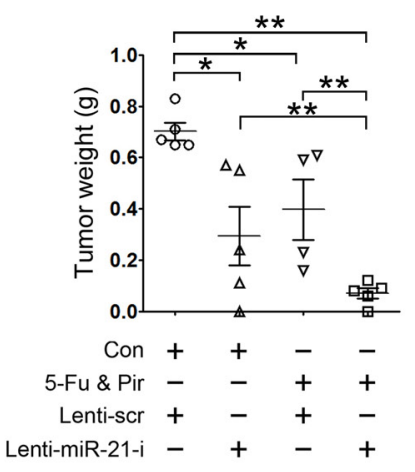

C
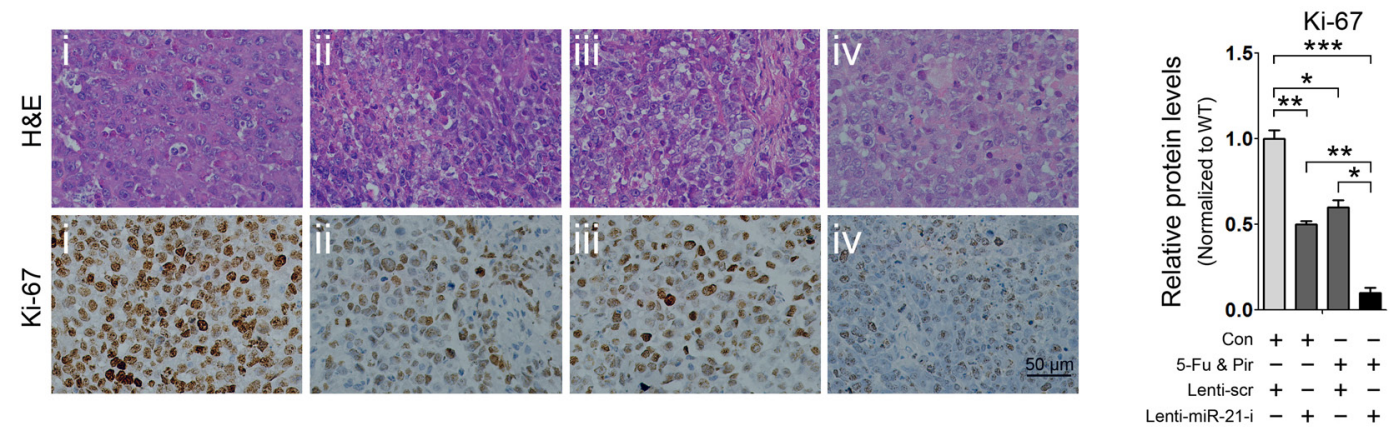

Figure 2: miR-21 suppression combined with 5-fluorouracil and pirarubicin treatment inhibits HCC xenograft growth. A and B: miR-21 inhibition in combination with 5-fluorouracil and pirarubicin treatment suppressed HCC xenograft growth. Representative photographs of nude mice (A, left panel) and photographs of dissected tumors from nude mice are shown (B, left panel). \# indicates no tumor formation. 5 mice in each group. Graphic representing the tumor volumes at the indicated days (A, right panel). Tumor weight was calculated at the end of the experiment (B, right panel). C: Pathology analysis of tissue sections from recipient mice at 4 weeks post-inoculation. H\&E staining and labeling with anti-Ki-67 was performed. Bars: $50 \mu \mathrm{m}$. 
A

B
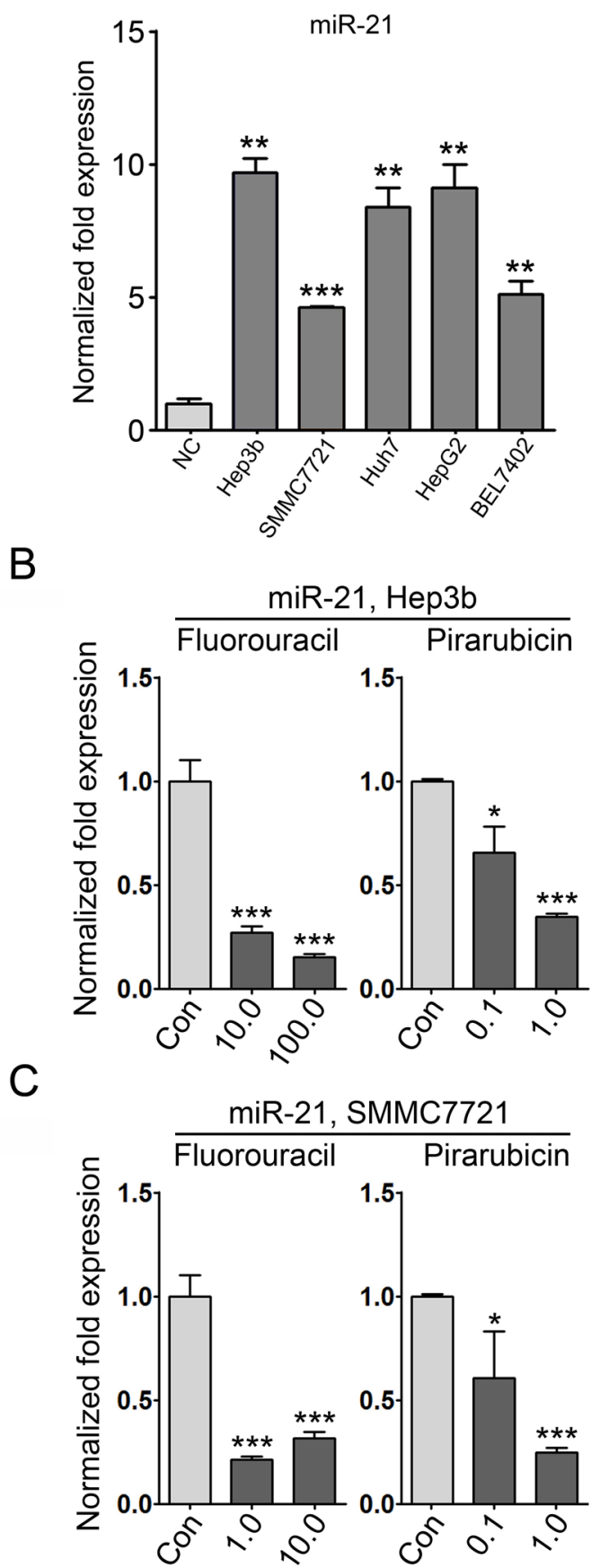

Pri-miR-21
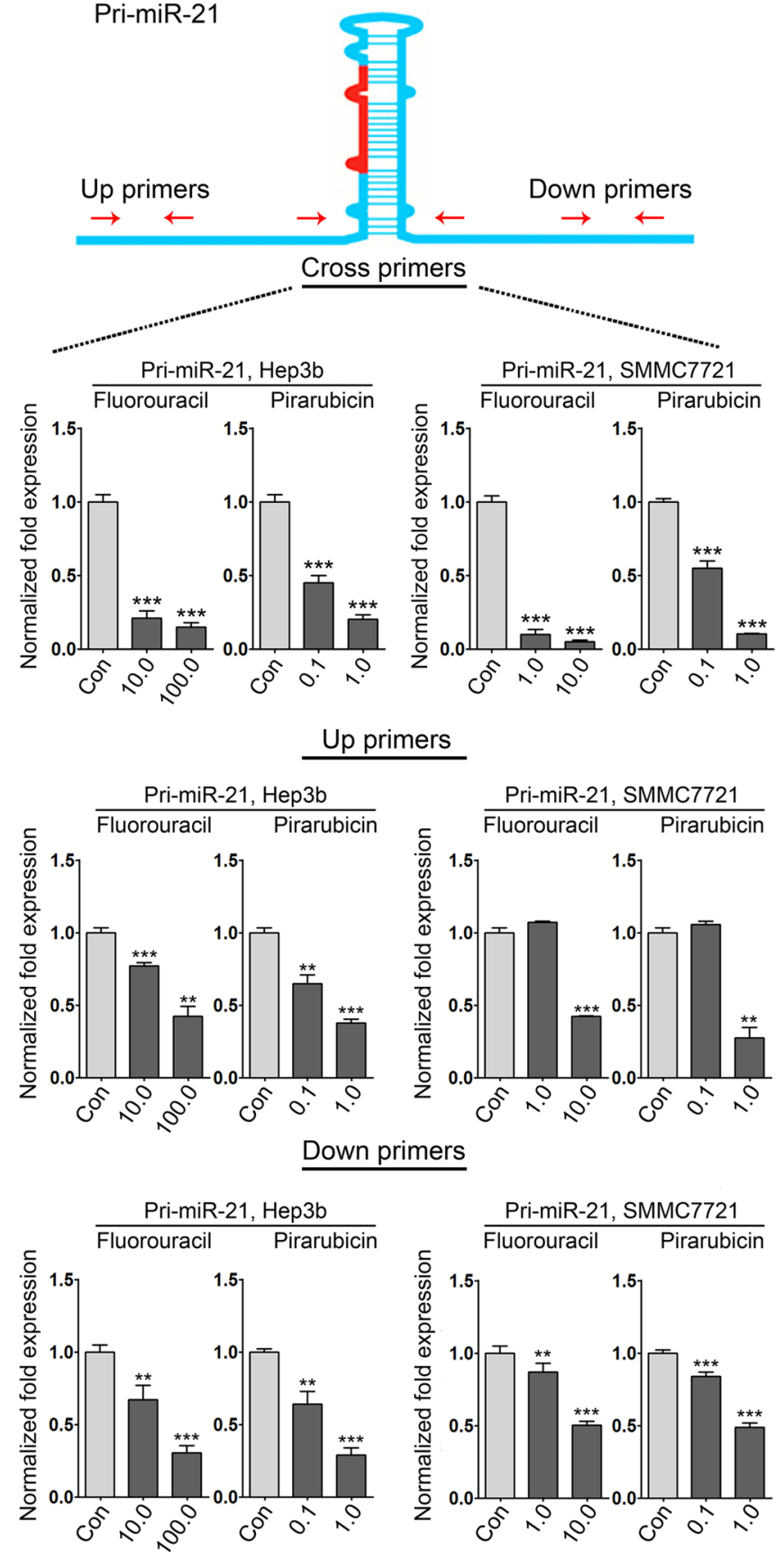

Figure 3: miR-21 expression is suppressed in HCC cells with 5-fluorouracil and pirarubicin treatment. A: qRT-PCR analysis was conducted to quantify endogenous miR-21 levels in normal liver (NC), Hep3b, SMMC7721, Huh7, HepG2, and Bel7402 cells. U6 was included as a control, and the data were normalized to the levels of NC. B and C: qRT-PCR analysis was conducted to quantify the expression of miR-21 in Hep3b (B) and SMMC7721 (C) cells following 5-fluorouracil and pirarubicin treatment. U6 was included as a control, and the data were normalized to control treated cells. D: Three pairs of primers were used in the qRT-PCR analysis to evaluate the expression of pri-miR-21 in Hep3b and SMMC7721 cells following 5-fluorouracil or pirarubicin treatment. GAPDH was included as a control, and the data were normalized to control cells. 
demonstrated similar results for the protein levels of JunB and c-Fos (Figure. 4D-i). Interestingly, we observed a marked decrease of p-c-Jun in HCC cells treated with 5-fluorouracil, but no significant change was found in c-Jun protein levels (Figure. 4D-ii). Similar results were found in pirarubicin treated HCC cells (Figure. 4E). Moreover, we evaluated the correlation between AP-1 components and miR-21 in HCC specimens. To this aim, 4 HCC specimens (miR-21-high, 2; miR-21-low, 2) were selected to determine the expression of miR-21 and AP-1 proteins. The analysis revealed that the HCC tissues with augmented AP-1 levels had increased miR-21 expression
(Figure. 4F; Figure. S4A). Further immunohistochemical analysis was performed in the same tissue pairs, and AP-1 staining was also increased in HCC tissues with high miR21 levels compared with the matched normal liver tissues (Figure. 4G; Figure. S4B).

Altogether, these data provide the evidence that 5-fluorouracil and pirarubicin have clinical benefits through modulating the AP-1/miR-21 axis in HCC.
A

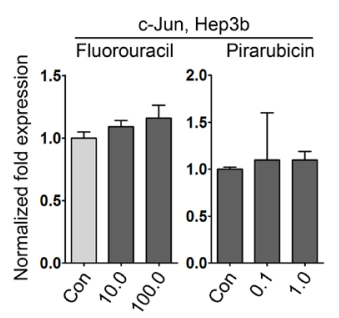

$\mathrm{B}$

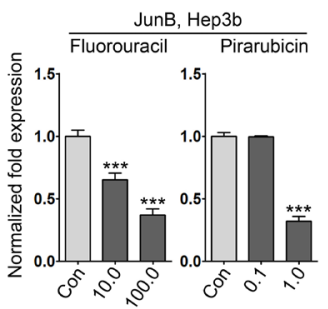

C
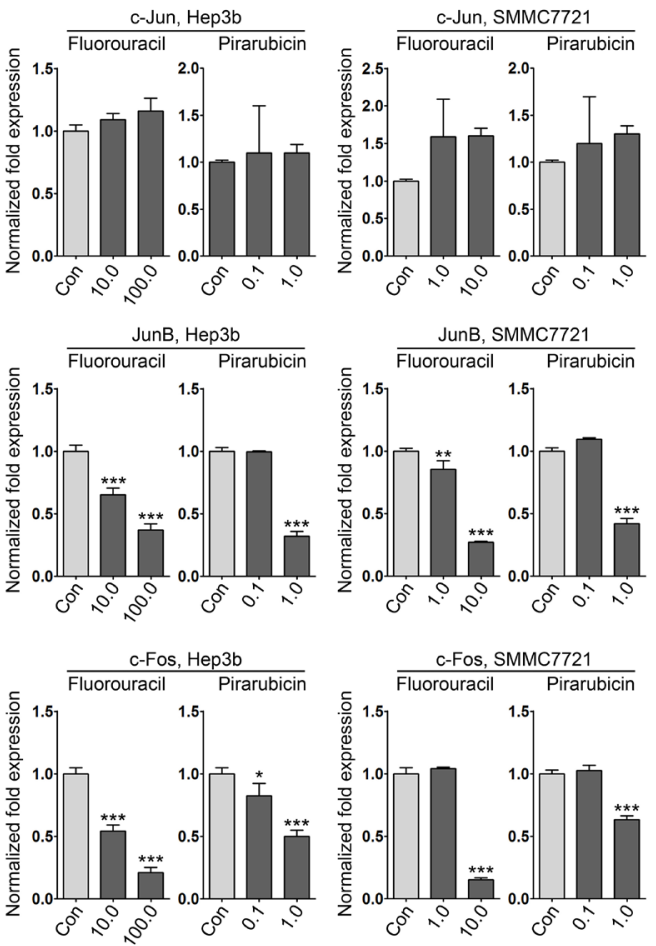

$\mathrm{F}$

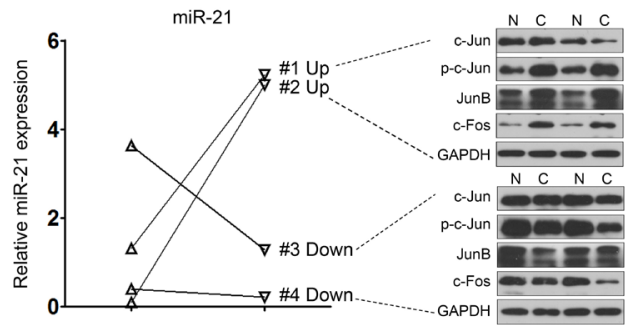

D

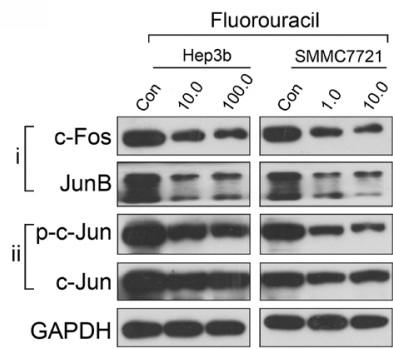

E

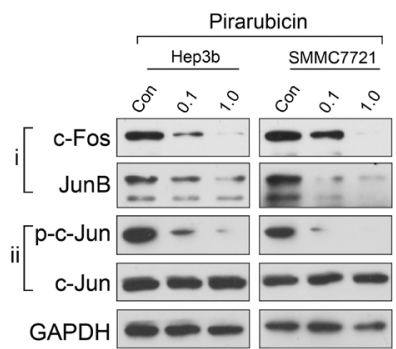

G
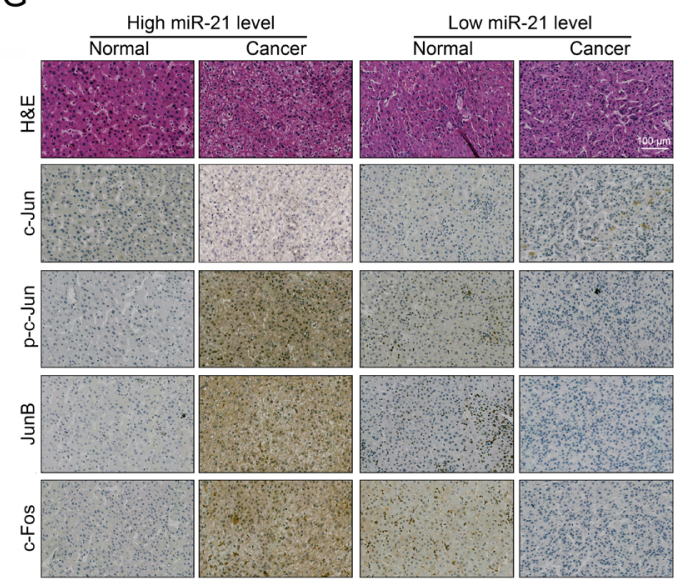

Figure 4: 5-fluorouracil and pirarubicin treatment leads to miR-21 downregulation in $\mathrm{HCC}$ cells via inhibiting the expression of AP-1 proteins. A, B, and C: qRT-PCR analysis was conducted to quantify the expression of AP-1 proteins (c-Jun, A; JunB, B; c-Fos, C) in Hep3b and SMMC7721 cells following 5-fluorouracil or pirarubicin treatment. GAPDH was included as a control, and the data were normalized to control cells. D and E: The protein level of AP-1 components in Hep3b and SMMC7721 cells after treatment with 5-fluorouracil (D) or pirarubicin (E) as measured through immunoblot analysis. GAPDH served as a loading control. F: qRT-PCR analysis of miR-21 expression in 4 pairs of HCC samples. U6 snRNA was used as a loading control. Immunoblot analysis of AP-1 proteins was performed using the same samples. G: Representative images of AP-1 proteins staining in the same samples described in $(\mathrm{F})$. Magnification is $20 \times$. Bars: $100 \mu \mathrm{m}$. 


\section{5-fluorouracil and pirarubicin suppress HCC cell growth via regulating the miR-21-mediated program}

Because an miR-21-mediated PTEN/PDCD4/RECK program has been identified in HepG2 and SMMC7721 HCC cells, we next investigated whether this program was modified by 5 -fluorouracil and pirarubicin treatment. The correlation between miR-21 and their targets was further validated in Hep3b cells, indicating that PTEN, PDCD4, and RECK were direct effectors of miR-21 in this cell line (Figure. 5A). We next conducted immunoblot analyses to evaluate the protein levels of PTEN, PDCD4, and RECK in Hep3b and SMMC7721 cells after 5-fluorouracil and pirarubicin treatment and we showed that the tested drugs increased the levels of these proteins, as compared to control-treated cells (Figure. 5B). To further probe the correlation between cell phenotypic alterations and drugsmediated AP-1/miR-21 axis, we performed a rescue assay that increased and then decreased the level of miR-21 targets via drugs treatment in combination with miR21 overexpression in $\mathrm{HCC}$ cells. Immunoblotting was used to evaluate PTEN, PDCD4, and RECK expression and demonstrated that the level of these proteins was altered under different treatment conditions in Hep3b and SMMC7721 cells (Figure. 5C). Furthermore, cell growth analysis was conducted to determine the alterations in cell viability corresponding with the expression level variations of targeted proteins, and we observed that miR-21 overexpression prevent the suppressive impact of 5-fluorouracil (Figure. 5D-i) and pirarubicin (Figure. 5Dii) treatment on $\mathrm{HCC}$ cell growth.

To further characterize the related genetic pathway affected by 5 -fluorouracil and pirarubicin involving the miR-21-mediated program in HCC cells, we examined the associated target genes including p-AKT, AKT, p-GSK3 $\beta$, GSK3 $\beta$, cyclin D1, cyclin E1, CDK2, and CDK4. Immunoblots were used to analyze the protein expression of these genes in Hep3b and SMMC7721 cells following 5 -fluorouracil and pirarubicin treatment. A decrease in p-AKT and p-GSK3 $\beta$ was noted in HCC cells treated with 5-fluorouracil compared with control-treated cells. However, no significant change was observed in total AKT and GSK $3 \beta$ levels. We also observed that cyclin D1, cyclin $\mathrm{E} 1, \mathrm{CDK} 2$, and CDK4 protein levels were decreased in 5-fluorouracil-treated HCC cells (Figure. 5E-i). Similar results were noted in $\mathrm{HCC}$ cells with pirarubicin treatment (Figure. 5E-ii).

Collectively, these findings indicate that chemotherapy has a clinical benefit against $\mathrm{HCC}$ through modifying the AP-1/miR-21-mediated axis (Figure. 5F).

\section{Drug-modulating oncomiRs or ts-miRs represent crucial mechanisms for HCC chemotherapy}

The above findings prompted us to further investigate the effect of 5 -fluorouracil and pirarubicin on other deregulated miRNAs validated in HCC. Hep3b and SMMC7721 cells were treated with these two drugs, and qRT-PCR was performed to measure the expression of selected oncomiRs and ts-miRs. After 5-fluorouracil and pirarubicin treatment, most ts-miRs were upregulated in HCC cells (17/18), and approximately $50 \%$ of the test oncomiRs were downregulated (8/16; Figure. 6A and Figure. S5). Importantly, miR-376a-P85 $\alpha$ and miR-122aBcl-w[29-30], which have been validated as deregulated axes in HCC cells, were modified after 5-fluorouracil and pirarubicin treatment, as shown by using qRT-PCR (Figure. 6B-C) and immunoblot analyses (Figure. 6D). These data suggested that miRNA-mediated programs modified by cytotoxic drugs may represent a global mechanism for HCC chemotherapy activity. In addition, the expression levels of several oncomiRs such as miR-205 and miR-221 were significantly induced by 5 -fluorouracil and pirarubicin treatment (Figure. S6), which might lead to chemoresistance in HCC.

Taken together, our findings suggest that multiple carcinogenesis-related programs, including oncomiR- or ts-miR-mediated axes, are involved in the $\mathrm{HCC}$ response to chemotherapy.

\section{DISCUSSION}

Increasing evidence clearly demonstrate that miR-21 not only is a crucial tumor promoter in hepatic tumorigenesis but also functions as a prognostic factor in HCC patients[21-22, 31]. Recent studies have also revealed the prognostic value of circulating miRNAs for detecting cancer[32]. For example, circulating miR21 serves as a biomarker for HCC diagnosis [23, 33]. However, the link between deregulated miR-21 and the prognosis of HCC patients undergoing HAIC has not been established. Here, we indicated that patients with higher miR-21 expression in tumors tended to relapse, which implied the prognostic significance of miR-21 for predicting the clinical benefit of HAIC with 5-fluorouracil and pirarubicin for $\mathrm{HCC}$ treatment. We also showed that HCC patients with a longer DFS tended to express lower miR-21 levels in tumors, albeit not to a significant degree $(P=0.055)$. The different clinical stages of the patients may contribute to this finding. Thus, HCC patients were divided into 2 groups, namely, early stage (pTNM I) and advanced stage (pTNM II-IV), based on our correlation analysis between miR-21 expression and pTNM stage. We observed that HCC patients at an early stage with lower miR-21 expression levels had a longer DFS after 5 -fluorouracil and pirarubicin treatment via HAIC. We 
A

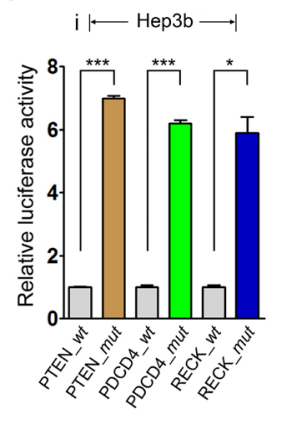

D
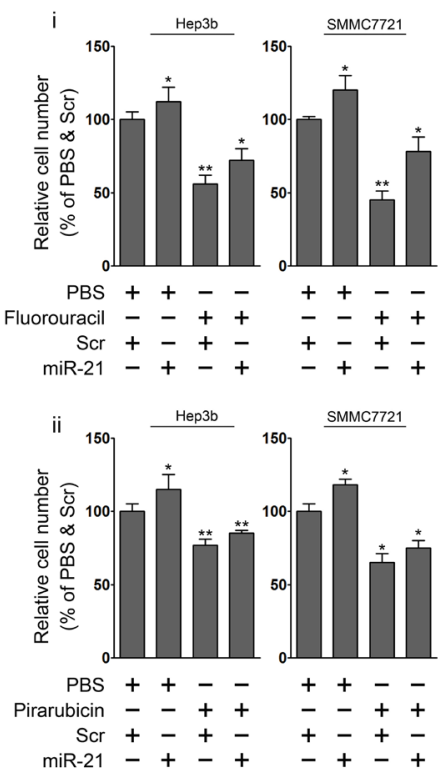

E



B
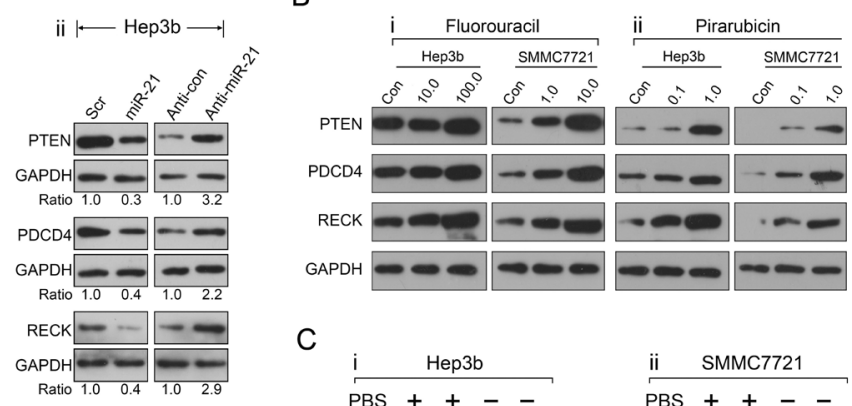

C

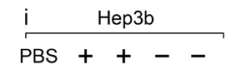

Fluorouracil -+++

$\mathrm{Scr}+-+-$

$\operatorname{miR}-21-+-+$

PTEN $=-$

\begin{tabular}{rrrrr} 
GAPDH & & & & \\
Ratio & & & & \\
\hline
\end{tabular}

\begin{tabular}{ccccc} 
Ratio 1.0 & 0.3 & 2.2 & 1.3 \\
\hline & - & &
\end{tabular}

PDCD4 - -

GAPDH $-\infty$

$\begin{array}{rrrr}\text { Ratio } 1.0 & 0.4 & 2.1 & 1.1 \\ \text { RECK } & - & & \end{array}$



$\begin{array}{llll}\text { Ratio } 10 & 0.6 & 22 & 17\end{array}$

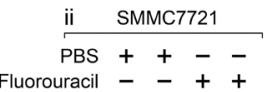

Fluorouracil -++

$\mathrm{Scr}+-+$

miR-21 -++

PTEN $=--$

GAPDH

$\begin{array}{lllll}\text { Ratio } 1.0 & 0.4 & 1.8 & 0.9\end{array}$

$\mathrm{PDCD} 4 \mathrm{-m}$

GAPDH -20

$\begin{array}{llll}\text { Ratio } 1.0 & 0.5 & 1.9 & 1.1\end{array}$

RECK - -

GAPDH

iii Hep3b

PBS ++-

Pirarubicin -++

$\mathrm{Scr}+-+$

miR-21 -++

PTEN --

GAPDH

$\begin{array}{lllll}\text { Ratio } & 0.4 & 1.5 & 0.7\end{array}$

$\mathrm{PDCD} 4--\cdots$

\begin{tabular}{rrrrr} 
GAPDH & & & & \\
Ratio 1.0 & 0.4 & 1.9 & 0.9 \\
\hline
\end{tabular}

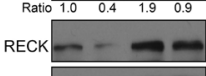

RECK - - -

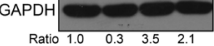

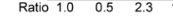

iv SMMC7721
PBS $++-\quad-$

Pirarubicin -++

$\mathrm{Scr}+-+$

miR-21 -+-+

PTEN - -

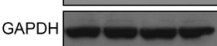

\begin{tabular}{lllll} 
Ratio 1.0 & 0.6 & 1.9 & 1.1 \\
\hline
\end{tabular}

$\mathrm{PDCD} 4 \longrightarrow-2$

GAPDH
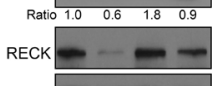

GAPDH

F



Figure 5: The miR-21-mediated program is modified by 5-fluorouracil and pirarubicin treatment in $\mathbf{H C C}$ cells. A: The correlation between miR-21 and its targets was validated in Hep3b cells via luciferase reporter assay (i) and immunoblots (ii) analysis. GAPDH was included as a loading control, and the data were normalized to scr or anti-control treated cells. The numbers below the panels represent the normalized protein expression levels. B: The protein levels of miR-21 target proteins in Hep3b and SMMC7721 cells after treatment with 5-fluorouracil (i) or pirarubicin (ii) as measured through immunoblot analysis. GAPDH served as a loading control. C: Modulation of miR-21 targets expression in 5-fluorouracil-treated Hep3b (i), SMMC7721 (ii) cells, and pirarubicin-treated Hep3b (iii), SMMC7721 (iv) cells was performed by transfection with miR-21 mimic. The target proteins level was measured through immunoblot analysis. GAPDH served as a loading control. The numbers below the panels represent the normalized protein expression levels. D: HCC cell growth was determined using CCK-8 at $36 \mathrm{~h}$ on different treatment conditions as described as (C). E: Immunoblot analyses of p-AKT, AKT, p-GSK-3 $\beta$, GSK-3 $\beta$, CDK2, and CDK4 were performed using Hep3b and SMMC7721 cells with 5-fluorouracil (i) and pirarubicin (ii) treatment. GAPDH served as the loading control. F: 5-fluorouracil and pirarubicin have clinical benefit for HCC treatment through modulating the AP-1 and miR-21-mediated axis. 
A

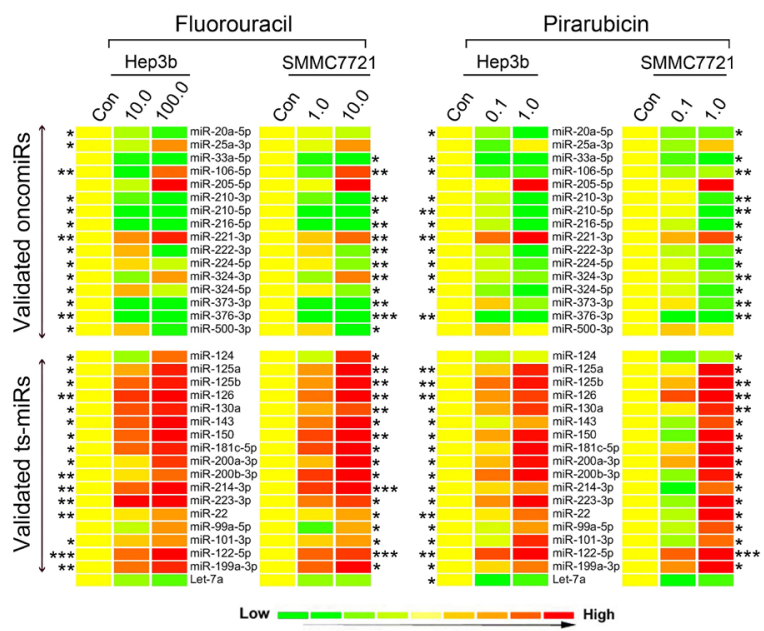

B

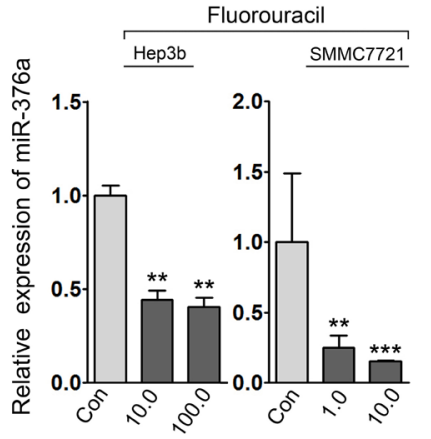

C
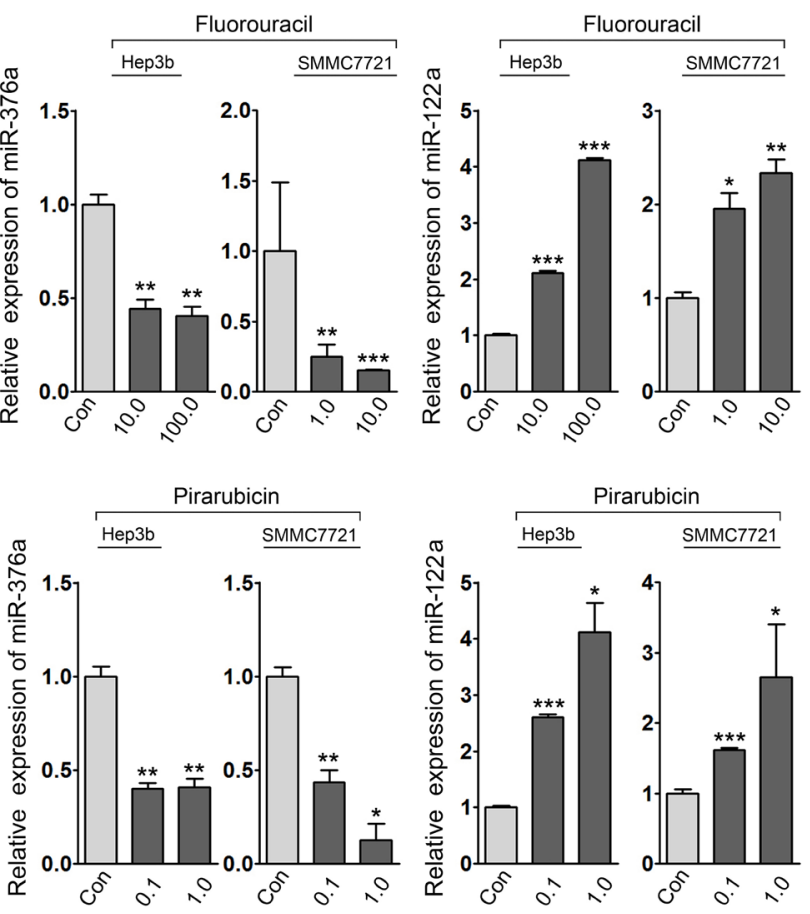

E

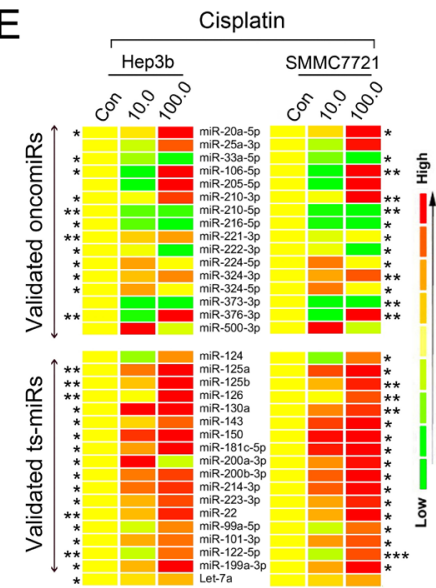

F
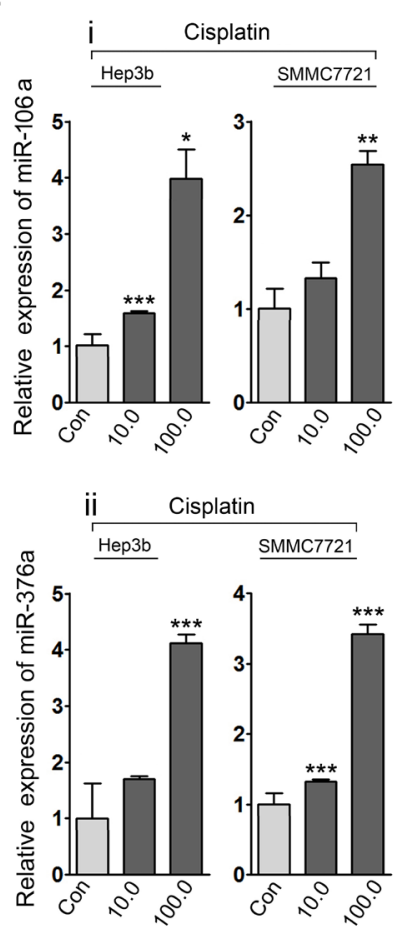

D
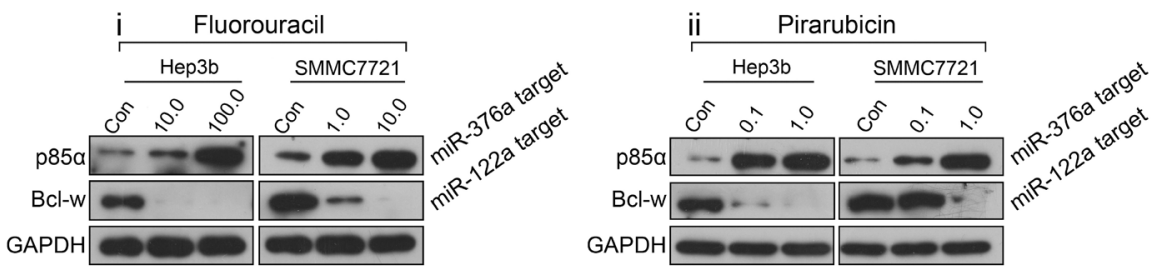

Figure 6: Drug-modulating oncomiRs or ts-miRs represent crucial mechanisms for HCC chemotherapy. A: qRT-PCR analysis quantified the expression of candidate oncomiRs and ts-miRs in Hep3b and SMMC7721 cells treated with 5-fluorouracil and pirarubicin. B and C: qRT-PCR analysis was performed to assess the expression of miR-376a and miR-122a in HCC cells treated with 5-fluorouracil (B) and pirarubicin (C). U6 snRNA served as loading controls. D: Immunoblot analysis was conducted to determine the expression of $\mathrm{p} 85 \alpha$ and Bcl-W in HCC cells treated with 5-fluorouracil (i) and pirarubicin (ii). GAPDH served as loading controls. E: qRT-PCR analysis quantified the expression of candidate oncomiRs and ts-miRs in Hep3b and SMMC7721 cells treated with cisplatin. F: qRT-PCR analysis was performed to assess the expression of miR-106a (i) and miR-376a (ii) in HCC cells treated with cisplatin. U6 snRNA served as loading controls. 
further generated subcutaneous xenografts by inoculating Hep3b cells with or without miR-21 inhibition to evaluate the antitumor effect of 5-fluorouracil and pirarubicin treatment in vivo, which indicated that miR-21 suppression in combination with 5-fluorouracil and pirarubicin treatment markedly inhibited HCC xenograft growth.

AP-1 activation plays a major role in tumor promotion and progression via regulating the expression of several oncogenes, which suggest that this transcriptional factors family might serve as a potential target for tumor therapy[34]. Several small molecular extracted from plant offer chemopreventive effects on tumorigenesis through inhibiting AP-1 activation, supporting this idea[35]. However, AP-1 is composed of a mixture of homo- and heterodimers of Jun and Fos proteins, which results in a complex phenotypic alteration of cells via regulating different target genes[36]. For example, ectopic expression of GADD153, which is also a putative target gene of AP1 , increases the sensitivity of MKN45 gastric cancer cells to VP-16, cisplatin, 5-fluorouracil, and docetaxel, indicating that augmented GADD153 expression and AP-1 binding activity promote apoptosis induced by anticancer drugs[37]. In the present study, we demonstrated that 5-fluorouracil and pirarubicin offered chemopreventive effects via inhibiting AP-1 activation, thereby leading to a modification of miR-21-mediated program in HCC cells. Our data suggest that the AP-1 pathway is a good target not only for the prevention but also for the therapy of HCC.

Cisplatin is another cytotoxic drugs widely used in HAIC for HCC treatment. We also investigated whether cisplatin exerted its suppressive effects on HCC cells via inhibiting miR-21 expression. Although several reports indicate that miR-21 inhibition lead to an improvement of cisplatin sensitivity[38-39], our qRT-PCR analysis revealed that cisplatin treated-Hep3b and SMMC7721 cells depicted no significant change in miR-21 levels (Figure. S7). These data suggest that the affected genetic pathways of cisplatin might be different with 5-fluorouracil and pirarubicin. We further conducted cisplatin treatment in HCC cells and performed expression analyses of some oncomiRs and ts-miRs tested in 5-fluorouracil and pirarubicin treatment. A different miRNA expression profile was observed in cisplatin treated cells compared with 5-fluorouracil and pirarubicin treatment (Figure. 6E-F). These findings represent a global mechanism for chemotherapy achieving clinical promise for HCC prevention through regulating miRNA-mediated axes but drugs may offer their effects through different genetic pathways.

Taken together, our findings indicate that HAIC prevention using 5-fluorouracil and pirarubicin has clinical promise for HCC patients through modulating the AP-1 and miR-21 axis. miR-21 could function as a potential biomarker for predicting the prognosis of HCC patients receiving HAIC treatment.

\section{MATERIALS AND METHODS}

\section{Human Liver Tissues and Cell Lines}

This study was approved by the ethical board of the Peking Union Medical College hospital and the ethical board of the Institute of Basic Medical Sciences, Chinese Academy of Medical Sciences, and all samples were obtained with patients' informed consent. Paired samples of tumor/non-tumor liver tissues were collected from patients undergoing HCC surgery, immediately snap frozen in liquid nitrogen, and stored at $-80^{\circ} \mathrm{C}$ until RNA extraction. The patient characteristics are provided in Supplementary Table 1. Follow-up studies were performed on $67 \mathrm{HCC}$ patients who underwent HAIC. The total RNA pool of normal liver samples comprised RNA from at least 3 donors, and the information for each healthy donor, including the age, sex, race, cause of death, and diagnosis, was obtained from Ambion Inc.

HCC cell lines, Hep3b, and SMMC7721 were purchased from the Shanghai Cell Bank, Chinese Academy of Sciences and cultured under standard conditions.

\section{RNA isolation and quantitative real-time PCR (qRT-PCR) analysis}

Total RNA was extracted from cells and tissues using Trizol (Invitrogen, Carlsbad, CA, USA) according to the manufacturer's instruction. The RNA was quantified by absorbance at $260 \mathrm{~nm}$. To assess the levels of miR-21, qRT-PCR analysis was conducted by using Taqman probes (Invitrogen, Carlsbad, CA, USA) in the Bio-Rad IQ5 qRTPCR system according to the manufacturer's instruction. The data were normalized using endogenous U6 snRNA. The expression levels of miRNAs in cancer relative to its non-tumorous control and $\mathrm{HCC}$ cells were calculated using the equation $2^{-\Delta \Delta C \mathrm{C}}$ in which $\Delta \mathrm{C}_{\mathrm{T}}=\mathrm{C}_{\mathrm{T}} 21-\mathrm{C}_{\mathrm{T}} \mathrm{U} 6$. The value of the relative expression ratio $<1.0$ was considered as low expression in cancer relative to the non-tumorous control, where others were considered as high expression. To assess the levels of pri-miR-21 and AP- 1 components in tested HCC cells, qRT-PCR analysis was conducted by using Taqman probes (Invitrogen, Carlsbad, CA, USA) in the Bio-Rad IQ5 qRT-PCR system according to the manufacturer's instruction. The data were normalized using endogenous GAPDH. Primers for qRT-PCR are shown in Supplementary Table 3.

\section{Constructs, reagents and assays}

The perfect complementary sequence of miR-21 and the 3'UTRs of the human PTEN, PDCD4, and RECK mRNA were cloned in between the Not1 and Xba1 sites 
of pRL-TK (Promega). Sequences of the other primers were shown in Supplementary Table 3. MiRNA mimics specifying miR-21 and control miRNA mimic were obtained from Dharmcon Inc. Cisplatin is a product of Hospira Austrila Pty Ltd., 5-fluorouracil is a product of Tianjin Kingyork Amino Acid Co., Ltd., and pirarubicin is a product of Shenzhen Main Luck Pharmaceuticals Inc.

Hep3b cells were seeded onto 24-well plates $\left(1 \times 10^{5}\right.$ cells per well) the day before transfections. Cells $(\approx 70 \%$ confluent) were transfected with pRL-TK luciferase reports (50 ng per well), pGL3-control firefly luciferase (10 ng per well). All transfections were carried out in triplicate with Effectene (QIAGEN). Cell lysates were prepared with Passive Lysis Buffer (Promega) $48 \mathrm{~h}$ after transfection, and luciferase activities were measured by using the Dual Luciferase Reporter Assay (Promega).

\section{Cell growth assay}

The cellular proliferation rate was measured using CCK-8 (DOJINDO) as previously described[21]. To measure the effects on cellular proliferation rates, cells were incubated in $10 \%$ CCK-8 (DOJINDO) diluted in normal culture media at $37^{\circ} \mathrm{C}$ until the appearance of visual color. Proliferation rates were determined at 12, 24, 36,48 , and $60 \mathrm{~h}$ post-transfection and quantification was done on a microtiter plate reader (Spectra Rainbow, Tecan) using the protocol recommended by the manufacturer.

\section{Western blots}

We extracted proteins from Hep3b, SMMC7721 cells, and HCC tissues with mammalian cell lysis buffer M-PER (PIERCE) containing protease and phosphatase inhibitor. Proteins from total cell lysates were resolved with a $4-20 \%$ Tris- $\mathrm{HCl}$ gradient gel (Bio-Rad), transferred to PVDF membranes, blocked in $5 \%$ nonfat milk or BSA in TBS/Tween-20, and blotted with antibodies for PTEN, PDCD4, RECK, c-Jun, JunB, c-Fos, p-AKT, AKT, p-GSK3 $\beta$, GSK3 $\beta$, cyclin D1, cyclin E1, CDK2, CDK4, and GAPDH (RECK and c-Jun from BD biosciences, cyclin D1, cyclin E1, CDK2, CDK4, and GAPDH from Abcam, others from CST).

\section{Immunohistochemistry}

HCC tissues and mouse tumor tissues were made into paraffin sections and pretreated at $65^{\circ} \mathrm{C}$ for 2 hours, followed by deparaffinization. Antigen retrieval was carried out before application of the primary antibodies (Ki-67, 1:100; DAKO), overnight at $4^{\circ} \mathrm{C}$. As a negative control, sections were incubated with normal IgG. Thereafter, slides were incubated for 2 hours at room temperature with the secondary antibody conjugated to horseradish peroxidase (HRP; 1:100; DAKO). HRP activity was detected using the Liquid DAB+ Substrate Chromogen System (DAKO). Finally, sections were counterstained with hematoxylin and photographed.

\section{Evaluation of immunohistochemical staining}

Immunohistochemistry stained sections were examined using an Olympus Vanox- $\mathrm{T}$ AH-2 light microscope (Olympus, Tokyo, Japan). The same magnification (microscope objective) was used to record all images in a particular series. Both the field limiting and contrast apertures were kept at the fully open position during (digital) photography to avoid any variability in reproducing aperture settings. Images were recorded with a Spot RTCCD camera (Diagnostic Instruments, Sterling Heights, MI, USA) in color mode, using full $(1600 \times 1200$ bit) resolution at 8-bit depth for each (RGB) color component. The photographs were taken with identical exposure settings. A flat field image was obtained at the beginning of each microscopy session and then used throughout the session to correct for uneven illumination. The white balance was adjusted for each slide. Recorded images were stored in color mode as uncompressed files in tagged image file format. The subsequent digital images were analyzed using the builtin functions of ImagePro Plus image analysis software 6.0 (Media Cybernetics, Silver Spring, MD, USA) by three pathologists simultaneously. Five fields of view were chosen stochastically by each pathologist. The procedure for determining the immunostaining intensity was as previously described[40].

\section{HCC xenograft model}

All animal procedures were performed according to the national Animal Experimentation guidelines (D.L.116/92) upon approval of the experimental protocol by the Institutional Animal Experimentation Committee of Peking Union Medical College. For the xenograft assay, six-week-old female nude mice (BALB/c-nude) were used to examine tumorigenicity. Hep3b HCC cells with or without miR-21 inhibition by using lentiviral system (Lenti-miR-21-I and Lenti-scr) were propagated $\left(6 \times 10^{6}\right.$ cells saline/Matrigel (BD Pharmigen San Jose, Ca), 1:1 v/v) and inoculated s.c. into the dorsal flanks of 20 mice ( 5 for each group as described). The size of the tumors was measured by caliper twice a week, and tumor volumes were calculated using the following formula: $\pi / 6 \times \mathrm{d}^{2} \times \mathrm{D}$ removed and weighed 7 weeks after tumor cell injection. Treatments were started after tumor reached approximately $100 \mathrm{~mm}^{3}$. 5-fluorouracil $\left(500 \mathrm{mg} / \mathrm{m}^{2}\right)$ and pirarubicin $\left(20 \mathrm{mg} / \mathrm{m}^{2}\right)$ were administered once a week, intravenously. Control mice received physiological saline only, according to the same schedule. 


\section{Statistics}

Each experiment was repeated at least 3 times. Student's $t$ test (2-tailed) was performed to compare 2 groups $(P<0.05$ was considered significant) unless otherwise indicated ( $\mathrm{X}^{2}$ test), and data for 3 groups were analyzed using 1-way analysis of variance. Correlations between miRNA expression and clinicopathological features were analyzed using non-parametric tests, such as the Mann-Whitney $U$-test for differences between 2 groups and the Kruskal-Wallis test for differences among 3 or more groups. All statistical analyses were performed using SPSS 16.0 software (SPSS Inc., Chicago, IL, USA). $P$-values $<0.05$ were considered significant.

\section{ACKNOWLEDGEMENTS}

We thank C. W Jia and Y. X Meng for assistance with the immunohistochemical analysis.

This research was supported by the National Nature Science Foundation of China (2011, 81100608, to C. Z. L.; 2014, 91129716, to X. D. H.), the IBMS, CAMS (2009PY13, 2010PYZ18, to C. Z. L.), the Beijing Nova Program (xx2012035 to Y.K.), and the Beijing Municipal Natural Science Foundation (7152033, to Y.K.).

\section{REFERENCES}

1. Ferlay J, Shin HR, Bray F, Forman D, Mathers C and Parkin DM. Estimates of worldwide burden of cancer in 2008: GLOBOCAN 2008. Int J Cancer. 2010; 127(12):28932917.

2. Jemal A, Bray F, Center MM, Ferlay J, Ward E and Forman D. Global cancer statistics. CA Cancer J Clin. 2011; 61(2):69-90.

3. Huang XH, Jian WH, Wu ZF, Zhao J, Wang H, Li W and Xia JT. Small interfering RNA (siRNA)-mediated knockdown of macrophage migration inhibitory factor (MIF) suppressed cyclin D1 expression and hepatocellular carcinoma cell proliferation. Oncotarget. 2014; 5(14):55705580 .

4. Takizawa D, Kakizaki S, Sohara N, Sato K, Takagi H, Arai H, Katakai K, Kojima A, Matsuzaki Y and Mori M. Hepatocellular carcinoma with portal vein tumor thrombosis: clinical characteristics, prognosis, and patient survival analysis. Dig Dis Sci. 2007; 52(11):3290-3295.

5. Cervello M, McCubrey JA, Cusimano A, Lampiasi N, Azzolina A and Montalto G. Targeted therapy for hepatocellular carcinoma: novel agents on the horizon. Oncotarget. 2012; 3(3):236-260.

6. Llovet JM and Bruix J. Systematic review of randomized trials for unresectable hepatocellular carcinoma: Chemoembolization improves survival. Hepatology. 2003; 37(2):429-442.
7. Reed ML, Vaitkevicius VK, Al-Sarraf M, Vaughn CB, Singhakowinta A, Sexon-Porte M, Izbicki R, Baker L and Straatsma GW. The practicality of chronic hepatic artery infusion therapy of primary and metastatic hepatic malignancies: ten-year results of 124 patients in a prospective protocol. Cancer. 1981; 47(2):402-409.

8. Terashima T, Yamashita T, Arai K, Sunagozaka H, Kitahara M, Nakagawa H, Kagaya T, Mizukoshi E, Honda M and Kaneko S. Feasibility and efficacy of hepatic arterial infusion chemotherapy for advanced hepatocellular carcinoma after sorafenib. Hepatol Res. 2013.

9. Xie Q, Su Y, Dykema K, Johnson J, Koeman J, De Giorgi V, Huang A, Schlegel R, Essenburg C, Kang L, Iwaya K, Seki S, Khoo SK, Zhang B, Buonaguro F, Marincola FM, et al. Overexpression of HGF Promotes HBV-Induced Hepatocellular Carcinoma Progression and Is an Effective Indicator for Met-Targeting Therapy. Genes Cancer. 2013; $4(7-8): 247-260$.

10. Farazi PA and DePinho RA. Hepatocellular carcinoma pathogenesis: from genes to environment. Nat Rev Cancer. 2006; 6(9):674-687.

11. Kasinski AL and Slack FJ. Epigenetics and genetics. MicroRNAs en route to the clinic: progress in validating and targeting microRNAs for cancer therapy. Nat Rev Cancer. 2011; 11(12):849-864.

12. Hatziapostolou M, Polytarchou C, Aggelidou E, Drakaki A, Poultsides GA, Jaeger SA, Ogata H, Karin M, Struhl K, Hadzopoulou-Cladaras M and Iliopoulos D. An HNF4alpha-miRNA inflammatory feedback circuit regulates hepatocellular oncogenesis. Cell. 2011; 147(6):1233-1247.

13. Varnholt $\mathrm{H}$. The role of microRNAs in primary liver cancer. Ann Hepatol. 2008; 7(2):104-113.

14. Kota J, Chivukula RR, O'Donnell KA, Wentzel EA, Montgomery CL, Hwang HW, Chang TC, Vivekanandan P, Torbenson M, Clark KR, Mendell JR and Mendell JT. Therapeutic microRNA delivery suppresses tumorigenesis in a murine liver cancer model. Cell. 2009; 137(6):10051017.

15. Yang H, Cho ME, Li TW, Peng H, Ko KS, Mato JM and Lu SC. MicroRNAs regulate methionine adenosyltransferase $1 \mathrm{~A}$ expression in hepatocellular carcinoma. J Clin Invest. 2013; 123(1):285-298.

16. van Kouwenhove M, Kedde M and Agami R. MicroRNA regulation by RNA-binding proteins and its implications for cancer. Nat Rev Cancer. 2011; 11(9):644-656.

17. Ge YY, Shi Q, Zheng ZY, Gong J, Zeng C, Yang J and Zhuang SM. MicroRNA-100 promotes the autophagy of hepatocellular carcinoma cells by inhibiting the expression of mTOR and IGF-1R. Oncotarget. 2014; 5(15):6218-6228.

18. Chai S, Tong M, Ng KY, Kwan PS, Chan YP, Fung TM, Lee TK, Wong N, Xie D, Yuan YF, Guan XY and Ma S. Regulatory role of miR-142-3p on the functional hepatic cancer stem cell marker CD133. Oncotarget. 2014; 
5(14):5725-5735.

19. Tsai WC, Hsu SD, Hsu CS, Lai TC, Chen SJ, Shen R, Huang Y, Chen HC, Lee CH, Tsai TF, Hsu MT, Wu JC, Huang HD, Shiao MS, Hsiao M and Tsou AP. MicroRNA-122 plays a critical role in liver homeostasis and hepatocarcinogenesis. J Clin Invest. 2012; 122(8):28842897.

20. Gramantieri L, Ferracin M, Fornari F, Veronese A, Sabbioni S, Liu CG, Calin GA, Giovannini C, Ferrazzi E, Grazi GL, Croce CM, Bolondi L and Negrini M. Cyclin G1 is a target of miR-122a, a microRNA frequently down-regulated in human hepatocellular carcinoma. Cancer Res. 2007; 67(13):6092-6099.

21. Liu C, Yu J, Yu S, Lavker RM, Cai L, Liu W, Yang K, He $\mathrm{X}$ and Chen S. MicroRNA-21 acts as an oncomir through multiple targets in human hepatocellular carcinoma. J Hepatol. 2010; 53(1):98-107.

22. Meng F, Henson R, Wehbe-Janek H, Ghoshal K, Jacob ST and Patel T. MicroRNA-21 regulates expression of the PTEN tumor suppressor gene in human hepatocellular cancer. Gastroenterology. 2007; 133(2):647-658.

23. Tomimaru Y, Eguchi H, Nagano H, Wada H, Kobayashi S, Marubashi S, Tanemura M, Tomokuni A, Takemasa I, Umeshita K, Kanto T, Doki Y and Mori M. Circulating microRNA-21 as a novel biomarker for hepatocellular carcinoma. J Hepatol. 2012; 56(1):167-175.

24. Tomimaru Y, Eguchi H, Nagano H, Wada H, Tomokuni A, Kobayashi S, Marubashi S, Takeda Y, Tanemura M, Umeshita K, Doki Y and Mori M. MicroRNA-21 induces resistance to the anti-tumour effect of interferon-alpha/5fluorouracil in hepatocellular carcinoma cells. Br J Cancer. 2010; 103(10):1617-1626.

25. Yamashita T, Arai K, Sunagozaka H, Ueda T, Terashima T, Mizukoshi E, Sakai A, Nakamoto Y, Honda M and Kaneko S. Randomized, phase II study comparing interferon combined with hepatic arterial infusion of fluorouracil plus cisplatin and fluorouracil alone in patients with advanced hepatocellular carcinoma. Oncology. 2011; 81(5-6):281290.

26. Zhang K, Chen J, Chen D, Huang J, Feng B, Han S, Chen Y, Song H, De W, Zhu Z, Wang R and Chen L. Aurora-A promotes chemoresistance in hepatocelluar carcinoma by targeting NF-kappaB/microRNA-21/PTEN signaling pathway. Oncotarget. 2014.

27. Talotta F, Cimmino A, Matarazzo MR, Casalino L, De Vita G, D'Esposito M, Di Lauro R and Verde P. An autoregulatory loop mediated by miR-21 and PDCD4 controls the AP-1 activity in RAS transformation. Oncogene. 2009; 28(1):73-84.

28. Misawa A, Katayama R, Koike S, Tomida A, Watanabe $\mathrm{T}$ and Fujita N. AP-1-Dependent miR-21 expression contributes to chemoresistance in cancer stem cell-like SP cells. Oncol Res. 2010; 19(1):23-33.

29. Lin CJ, Gong HY, Tseng HC, Wang WL and Wu JL.
miR-122 targets an anti-apoptotic gene, Bcl-w, in human hepatocellular carcinoma cell lines. Biochem Biophys Res Commun. 2008; 375(3):315-320.

30. Zheng Y, Yin L, Chen H, Yang S, Pan C, Lu S, Miao M and Jiao B. miR-376a suppresses proliferation and induces apoptosis in hepatocellular carcinoma. FEBS Lett. 2012; 586(16):2396-2403.

31. Karakatsanis A, Papaconstantinou I, Gazouli M, Lyberopoulou A, Polymeneas G and Voros D. Expression of microRNAs, miR-21, miR-31, miR-122, miR-145, miR-146a, miR-200c, miR-221, miR-222, and miR-223 in patients with hepatocellular carcinoma or intrahepatic cholangiocarcinoma and its prognostic significance. Mol Carcinog. 2013; 52(4):297-303.

32. Leidinger P, Backes C, Dahmke IN, Galata V, Huwer H, Stehle I, Bals R, Keller A and Meese E. What makes a blood cell based miRNA expression pattern disease specific? - A miRNome analysis of blood cell subsets in lung cancer patients and healthy controls. Oncotarget. 2014; 5(19):9484-9497.

33. Xu J, Wu C, Che X, Wang L, Yu D, Zhang T, Huang L, Li H, Tan W, Wang C and Lin D. Circulating microRNAs, miR-21, miR-122, and miR-223, in patients with hepatocellular carcinoma or chronic hepatitis. Mol Carcinog. 2011; 50(2):136-142.

34. Shaulian E and Karin M. AP-1 in cell proliferation and survival. Oncogene. 2001; 20(19):2390-2400.

35. Aggarwal BB, Takada $\mathrm{Y}$ and Oommen OV. From chemoprevention to chemotherapy: common targets and common goals. Expert Opin Investig Drugs. 2004; 13(10):1327-1338.

36. Kim R, Tanabe K, Emi M, Uchida Y, Inoue H and Toge T. Inducing cancer cell death by targeting transcription factors. Anticancer Drugs. 2003; 14(1):3-11.

37. Kim R, Ohi $\mathrm{Y}$, Inoue $\mathrm{H}$, Aogi $\mathrm{K}$ and Toge $\mathrm{T}$. Introduction of gadd153 gene into gastric cancer cells can modulate sensitivity to anticancer agents in association with apoptosis. Anticancer Res. 1999; 19(3A):1779-1783.

38. Chan JK, Blansit K, Kiet T, Sherman A, Wong G, Earle C and Bourguignon LY. The inhibition of miR-21 promotes apoptosis and chemosensitivity in ovarian cancer. Gynecol Oncol. 2014; 132(3):739-744.

39. Yang SM, Huang C, Li XF, Yu MZ, He Y and Li J. miR21 confers cisplatin resistance in gastric cancer cells by regulating PTEN. Toxicology. 2013; 306:162-168.

40. Paizs M, Engelhardt JI and Siklos L. Quantitative assessment of relative changes of immunohistochemical staining by light microscopy in specified anatomical regions. J Microsc. 2009; 234(1):103-112. 